\title{
Factors associated with emergency department presentation after total joint arthroplasty: a population-based retrospective cohort study
}

\author{
Bheeshma Ravi MD PhD, Timothy Leroux MD MEd, Peter C. Austin PhD, J. Michael Paterson MSc, \\ Suriya Aktar MSc, Donald A. Redelmeier MD MS(HSR)
}

\section{Abstract}

Background: Unplanned visits to the emergency department after total joint arthroplasty are far more common than unplanned readmissions. Our objectives were to characterize the prevalence of presentation to an emergency department for any reason after total joint arthroplasty and to identify risk factors for such visits.

\begin{abstract}
Methods: Using health administrative databases, we conducted a population-based retrospective cohort study of adults (19-89 yr of age) who received their first primary elective total hip arthroplasty (THA) or total knee arthroplasty (TKA) procedure for arthritis between April 2011 and March 2016 in Ontario. We made univariate comparisons between patients who presented to the emergency department within 30 days of surgery and those who did not in. We determined differences in use of health care services between groups by comparing the change in use in the year before and after surgery between patients who presented to the emergency department and those who did not. We developed logistic regression models for the occurrence of an emergency department visit using backward variable elimination.
\end{abstract}

Results: We identified 42273 total hip recipients and 70725 total knee recipients, of whom 5640 (13.3\%) and 11224 (15.9\%), respectively, presented to the emergency department within 30 days of surgery. Fewer than $1 \%$ of these patients required admission, and nearly half $(45 \%)$ went to a different institution from where they had their surgery. Among both THA and TKA recipients, patients who presented to the emergency department had a net increase in their median annual health care costs (THA: \$501, TKA: \$682), compared to a net decrease for the cohort as a whole. Factors associated with increased risk of an emergency visit included increased patient age, male sex, rural residence and various comorbidities. Predictive regression models showed poor discriminative ability for both THA (C-statistic 0.57 ) and TKA (C-statistic 0.58$)$ recipients.

Interpretation: One in 7 patients presented to the emergency department within 30 days of THA or TKA. Some may conceivably have been managed remotely, and very few required readmission. There is a crucial need for strategies to minimize these events.

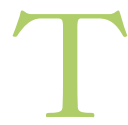

he recent shift toward a bundled payment for total joint arthroplasty has provided incentives for hospitals to reduce costs and minimize complications. A large priority has been on limiting hospital readmissions, which is one way that payers can also monitor the quality of care. ${ }^{1}$ However, hospital readmission after total joint arthroplasty is rare. ${ }^{2} \mathrm{~A}$ far less discussed but potentially more frequent event is an unplanned visit to an emergency department.

Recent studies suggest that the number of unplanned visits to the emergency department within 30 days of total joint arthroplasty is substantial and that such visits are expensive. ${ }^{3,4}$ However, these studies are mainly based on US data and did not identify emergency department visits to other centres, thereby potentially underestimating the problem. Each emergency department visit in Ontario costs about $\$ 400$, excluding physician costs. ${ }^{5}$ However, despite being costly and resource intensive, emergency department visits may not result in readmission. Preventable emergency department visits, therefore, are an important target for potential cost savings.

A better understanding of factors associated with postoperative emergency department presentation may help identify patients who may benefit from special intervention. To this end, we examined a large diverse cohort of patients from Ontario undergoing primary total hip arthroplasty (THA) or

\section{Competing interests: None declared.}

This article has been peer reviewed.

Correspondence to: Bheeshma Ravi, bheeshma.ravi@sunnybrook.ca CMAJ Open 2020. DOI:10.9778/cmajo.20190116 
total knee arthroplasty (TKA). Our objectives were to characterize the prevalence of 30-day emergency department presentation following joint replacement surgery and to identify characteristics associated with increased risk of presentation to the emergency department.

\section{Methods}

\section{Setting and study sample}

We defined a cohort of adults (19-89 yr of age) who received their first primary unilateral elective THA or TKA procedure for arthritis (degenerative or inflammatory) in Ontario between Apr. 1, 2011, and Mar. 31, 2016. ${ }^{6}$ We excluded patients with an acute length of stay greater than 7 days, as this was greater than the 95 th percentile for length of stay for our cohort and likely represents a complex condition. We also excluded patients whose primary address was outside the province at the time of surgery (i.e., an out-of-province claim) and those who emigrated out of the province within a year of surgery. We identified every procedure included in the study through a combination of physician billing claims for a joint replacement and hospital admission records for the same procedure.

\section{Data sources}

We used hospital discharge abstracts from the Canadian Institute for Health Information Discharge Abstract Database and physician claims from the Ontario Health Insurance Plan. We identified patients using specific procedure and diagnostic codes from the International Statistical Classification of Diseases and Related Health Problems, 10th Revision, enhanced Canadian version and the Canadian Classification of Health Interventions (THA: 1VA53LAPN/1VA53LLPN/1VA53PNPN and R440/R553; TKA: 1VG53LAPN/1VG53LAPP and R441/ R248). ${ }^{6}$ We accessed patient demographic characteristics via the Ontario Health Insurance Plan Registered Persons Database. ${ }^{7-9}$ We identified emergency department visits for any reason from the National Ambulatory Care Reporting System database. Each record in the administrative databases was linked by a unique individual identification number to identify the totality of a patient's interactions throughout the singlepayer health care system during the study.

\section{Outcomes}

Our primary outcome was a visit to any emergency department in Ontario for any reason within 30 days following surgery. Secondary outcomes included determining whether the emergency department was affiliated with the institution that provided the surgery and the reason for the visit. We identified the latter using the diagnoses recorded by the emergency physician. We categorized the most responsible diagnosis into the following categories: wound issues, concerns about infection, acute pain, cardiovascular diagnoses (e.g., possible congestive heart failure), gastrointestinal diagnoses (e.g., constipation), possible thrombophlebitis, familypractice-sensitive conditions (e.g., lichen planus, urticaria), genitourinary disorders (e.g., painful micturition), preexisting medical issues (e.g., exacerbation of type 2 diabetes mellitus), reaction to medications (e.g., opioid overdose) and miscellaneous. ${ }^{10}$ Family-practice-sensitive conditions are previously identified conditions for which visits to emergency departments are unnecessary because they have less than $1 \%$ chance of resulting in hospital admission and can be appropriately managed at a family physician's office. ${ }^{10}$

\section{Covariates}

We considered several factors that potentially influence the risk of complications following joint replacement, including patient age, sex, socioeconomic status and comorbidities. Using preexisting algorithms that use physician billing codes and hospital admission codes, we identified patients with a history of asthma, ${ }^{11}$ coronary artery disease, ${ }^{12}$ congestive heart failure, diabetes ${ }^{13}$ hypertension ${ }^{14}$ or chronic obstructive pulmonary disease ${ }^{15}$ (Appendix 1, available at www.cmajopen.ca/ content/8/1/E26/suppl/DC1). We used physician billing codes to identify patients who had previously received counselling regarding smoking cessation (E079, K039, Q042A).

We categorized comorbidities listed on hospital discharge abstracts in the 3 years before the index total joint arthroplasty admission according to an adaptation of the Charlson Comorbidity Index. ${ }^{16,17}$ We used Adjusted Clinical Groups (ACGs) based on diagnosis codes from hospital admissions and physician visits in the 2 years before surgery to define "frail" patients (Johns Hopkins ACG System Version 10.0)..$^{18}$ Among THA recipients, we used physician billing codes to identify patients with a body mass index greater than 40 ; these codes are not available for TKA recipients. ${ }^{19}$

We included 2 validated measures of socioeconomic status: the neighbourhood income quintile and the Ontario Marginalization Index. Neighbourhood income quintiles categorize small geographic areas into 5 roughly equal population groups, with the lowest quintile being the least affluent neighbourhoods..$^{20,21}$ The Ontario Marginalization Index is an Ontario-specific version of the Canadian Marginalization Index. It has been shown to be stable across time periods and across different geographic areas. ${ }^{22}$ It comprises 4 elements: ethnic concentration, residential instability, dependency and deprivation. ${ }^{23}$ Each element is sorted into quintiles, arranged from least (first quintile) to most (fifth quintile) marginalized.

For each patient, we defined the corresponding surgeon volume as the number of joint-specific (hip or knee) arthroplasty procedures (both primary and revision) performed by the primary surgeon in the 365 days before the index procedure. We similarly defined hospital volume at the institutional level. We identified use of general anesthesia from the hospital record. We determined direct health care costs in the year before and after surgery using previously established methods for case costing in Ontario's administrative databases. ${ }^{24}$

\section{Statistical analysis}

All analyses were stratified by the joint being replaced (hip or knee). We made univariate comparisons between patients who presented to the emergency department and those who did not. We then developed logistic regression models for the 
occurrence of an emergency department visit within 30 days of discharge. We used backward variable elimination to create a parsimonious regression model using the following candidate predictor variables: patient demographic characteristics (age, sex, income quintile, Ontario Marginalization Index, living in a rural area), comorbidities (Charlson Comorbidity Index score, frailty, asthma, chronic obstructive pulmonary disease, coronary artery disease, congestive heart failure, receiving counselling about smoking cessation, diabetes, hypertension, morbid obesity [THA only]) and provider characteristics (hospital volume, surgeon volume). ${ }^{25}$ The type I error probability was set to 0.05 for all analyses.

\section{Cost analysis}

Arthroplasty has previously been established to result in an almost immediate reduction in annual use of health care services. ${ }^{26,27}$ However, this potential saving may be offset by the costs of visiting the emergency department after surgery. To assess medical costs incurred by our cohort, we used data from the Ontario Ministry of Health and Long-Term Care ${ }^{28-34}$ to determine public medical costs in the year before and after admission for arthroplasty. We assigned costs to each patient based on the year when they were incurred and then standardized them to 2013 Canadian dollars using the health care component of the Ontario Consumer Price Index (www. statscan.gc.ca). We compared changes in annual use of health care services between groups using the differences-indifferences technique. To do so, we deducted costs in the year before surgery from the costs accrued in the year following the arthroplasty among patients who did not present to the emergency department (first difference). We then compared this difference to the same difference among patients who did present to the emergency department (second difference). ${ }^{24}$

\section{Ethics approval}

This study was completed with administrative data from ICES that included a waiver for institutional review board approval. Use of the data in this study was authorized under section 45 of Ontario's Personal Health Information Protection Act.

\section{Results}

We identified 42273 eligible THA and 70725 eligible TKA recipients between Apr. 1, 2011, and Mar. 31, 2016, of whom $5640(13.3 \%)$ and 11224 (15.9\%), respectively, had an emergency department visit within 30 days of surgery (Tables 1 and 2). Among patients who visited the emergency department, 3014 (53.4\%) of THA recipients and 6263 (55.8\%) TKA recipients presented at the same hospital where they had their surgery. Among both THA and TKA recipients, patients who presented to the emergency department had a net increase in their median annual health care costs (THA: \$501; TKA: \$682), compared to a net decrease in the cohort as a whole. The readmission rate for patients who presented to an emergency department was $1.0 \%$ and $0.6 \%$ in the THA and TKA cohorts, respectively.

\section{Types of emergency}

We found 588 and 751 unique diagnoses among THA and TKA recipients, respectively. Concerns over the surgical wound, possible infection and acute pain constituted the majority of diagnoses for both groups (Table 3).

\section{Multivariable models for emergency visits}

Among THA recipients, the multivariable model consisted of patient age (> $70 \mathrm{yr}$ ), male sex, rural residence and specific comorbidities (chronic obstructive pulmonary disease, obesity, hypertension) (Table 4). The C-statistic was 0.571 for this model.

Among TKA recipients, the multivariable model consisted of patient age $(<50 \mathrm{yr}$ or $>70 \mathrm{yr})$, male sex, rural residence, lowest neighbourhood income quintile and specific comorbidities (chronic obstructive pulmonary disease, congestive heart failure, asthma, complicated diabetes) (Table 4). The C-statistic for this model was 0.578 .

\section{Interpretation}

In a large cohort of patients who underwent joint replacement in Ontario, we found that 1 in 7 presented to the emergency department within 30 days of surgery. The most common reasons were wound issues, concerns about infection and acute pain. Fewer than $1 \%$ of patients required admission. Almost half (about $45 \%$ ) presented to a different institution from where their surgery was done. Factors associated with increased risk for an emergency department visit included patient age, male sex, rural residence and various comorbidities.

Although the rate of presentation to the emergency department was high, most patients could be managed as outpatients. This suggests the need to educate patients on the normal course of pain and wound appearance after total joint arthroplasty. However, reduced hospital lengths of stay may lead to fewer opportunities for this education. Many hospitals provide patients with teaching preoperatively and also provide written materials detailing the normal postoperative course. However, our results suggest that more education is needed. Another option that could be used is electronic communication after discharge, including the ability to send pictures of the surgical wound to the primary surgeon, although this would need to be implemented in a manner that safeguards patient privacy. ${ }^{35,36}$

Emergency department visits often result in unnecessary testing (e.g., ultrasonography to rule out deep vein thrombosis) or unnecessary treatment (e.g., oral antibiotic therapy for possible infection). Unnecessary investigations and treatments not only expose patients to additional risk (e.g., use of antibiotics), but are also costly. ${ }^{37}$ Although most arthroplasty recipients showed a net decrease in annual health care costs after their surgery, patients who presented to the emergency department had a net increase in costs, despite the lack of readmission or additional surgery for most.

The strongest predictor of an emergency visit in both cohorts was rural residence. Patients who live outside cities have fewer local options for urgent issues. Patients typically 
Table 1: Characteristics of eligible total hip arthroplasty recipients

\begin{tabular}{|c|c|c|c|c|}
\hline \multirow[b]{2}{*}{ Characteristic } & \multicolumn{3}{|c|}{ No. $(\%)$ of patients* } & \multirow[b]{2}{*}{$\begin{array}{l}\text { Standardizec } \\
\text { difference }\end{array}$} \\
\hline & $\begin{array}{l}\text { All patients } \\
n=42273\end{array}$ & $\begin{array}{c}\text { Emergency } \\
\text { department visit } \\
n=5640\end{array}$ & $\begin{array}{c}\text { No emergency } \\
\text { department visit } \\
n=36633\end{array}$ & \\
\hline Age, yr, median (IQR) & $67(59-74)$ & $68(60-76)$ & $67(59-74)$ & 0.12 \\
\hline \multicolumn{5}{|l|}{ Sex } \\
\hline Female & 22758 (53.8) & $2881(51.1)$ & 19877 (54.3) & 0.06 \\
\hline Male & $19515(46.2)$ & $2759(48.9)$ & $16756(45.7)$ & 0.06 \\
\hline Rural residence & $6956(16.5)$ & $1218(21.6)$ & $5738(15.7)$ & 0.15 \\
\hline \multicolumn{5}{|l|}{ Ethnic concentration quintile†‡ } \\
\hline 1 (lowest) & $10601(25.2)$ & $1595(28.5)$ & $9006(24.7)$ & 0.09 \\
\hline 2 & $9968(23.7)$ & $1324(23.7)$ & $8644(23.7)$ & 0.0 \\
\hline 3 & $8988(21.4)$ & 1087 (19.4) & $7901(21.7)$ & 0.06 \\
\hline 4 & $7524(17.9)$ & $945(16.9)$ & $6579(18.1)$ & 0.03 \\
\hline 5 (highest) & $4961(11.8)$ & $643(11.5)$ & $4318(11.8)$ & 0.01 \\
\hline \multicolumn{5}{|l|}{ Instability quintile†‡ } \\
\hline 1 (lowest) & $6457(15.4)$ & $731(13.1)$ & $5726(15.7)$ & 0.08 \\
\hline 2 & 8589 (20.4) & $1063(19.0)$ & $7526(20.6)$ & 0.04 \\
\hline 3 & 9031 (21.5) & $1223(21.9)$ & 7808 (21.4) & 0.01 \\
\hline 4 & $8673(20.6)$ & $1265(22.6)$ & $7408(20.3)$ & 0.06 \\
\hline 5 (highest) & $9292(22.1)$ & $1312(23.5)$ & $7980(21.9)$ & 0.04 \\
\hline \multicolumn{5}{|l|}{ Dependency quintile†‡ } \\
\hline 1 (lowest) & $5771(13.7)$ & $744(13.3)$ & $5027(13.8)$ & 0.01 \\
\hline 2 & $7018(16.7)$ & $890(15.9)$ & $6128(16.8)$ & 0.02 \\
\hline 3 & $7945(18.9)$ & $977(17.5)$ & $6968(19.1)$ & 0.04 \\
\hline 4 & $8714(20.7)$ & $1150(20.6)$ & $7564(20.8)$ & 0.0 \\
\hline 5 (highest) & $12594(30.0)$ & $1833(32.8)$ & $10761(29.5)$ & 0.07 \\
\hline \multicolumn{5}{|l|}{ Deprivation quintile†‡ } \\
\hline 1 (lowest) & $10144(24.1)$ & $1219(21.8)$ & $8925(24.5)$ & 0.06 \\
\hline 2 & $9217(21.9)$ & $1203(21.5)$ & $8014(22.0)$ & 0.01 \\
\hline 3 & 8375 (19.9) & $1151(20.6)$ & $7224(19.8)$ & 0.02 \\
\hline 4 & 7845 (18.7) & $1091(19.5)$ & $6754(18.5)$ & 0.02 \\
\hline 5 (highest) & $6461(15.4)$ & $930(16.6)$ & $5531(15.2)$ & 0.04 \\
\hline \multicolumn{5}{|l|}{ Income quintileł } \\
\hline 1 (lowest) & 6475 (15.3) & $964(17.1)$ & $5511(15.1)$ & 0.06 \\
\hline 2 & $7861(18.6)$ & $1072(19.1)$ & $6789(18.6)$ & 0.01 \\
\hline 3 & $8446(20.0)$ & $1135(20.2)$ & $7311(20.0)$ & 0.0 \\
\hline 4 & $8899(21.1)$ & $1173(20.8)$ & $7726(21.1)$ & 0.01 \\
\hline 5 (highest) & $10510(24.9)$ & $1282(22.8)$ & $9228(25.2)$ & 0.06 \\
\hline \multicolumn{5}{|l|}{ Comorbidities } \\
\hline Asthma & $1483(3.5)$ & $252(4.5)$ & $1231(3.4)$ & 0.06 \\
\hline Coronary artery disease & $1358(3.2)$ & $220(3.9)$ & $1138(3.1)$ & 0.05 \\
\hline Congestive heart failure & $1773(4.2)$ & $305(5.4)$ & $1468(4.0)$ & 0.07 \\
\hline Chronic obstructive pulmonary disease & $7304(17.3)$ & $1186(21.0)$ & $6118(16.7)$ & 0.11 \\
\hline Counselled about smoking cessation & $2560(6.1)$ & $351(6.2)$ & $2209(6.0)$ & 0.01 \\
\hline Diabetes & $8396(19.9)$ & $1219(21.6)$ & $7177(19.6)$ & 0.05 \\
\hline Complicated diabetes & $1553(3.7)$ & $261(4.6)$ & $1292(3.5)$ & 0.06 \\
\hline Hypertension & 26723 (63.2) & $3768(66.8)$ & 22955 (62.7) & 0.09 \\
\hline Frailty & $2077(4.9)$ & $309(5.5)$ & $1768(4.8)$ & 0.03 \\
\hline Body mass index $>40$ & $2462(5.8)$ & $391(6.9)$ & $2071(5.7)$ & 0.05 \\
\hline \multicolumn{5}{|l|}{ Charlson Comorbidity Index score } \\
\hline 0 & $32511(76.9)$ & $4196(74.4)$ & $28315(77.3)$ & 0.07 \\
\hline 1 & $6141(14.5)$ & $832(14.8)$ & $5309(14.5)$ & 0.01 \\
\hline 2 & $2344(5.5)$ & $403(7.1)$ & $1941(5.3)$ & 0.08 \\
\hline$\geq 3$ & $1277(3.0)$ & $209(3.7)$ & $1068(2.9)$ & 0.04 \\
\hline \multicolumn{5}{|l|}{ Admission characteristics } \\
\hline Hospital volume, median (IQR) & $277(195-515)$ & $274(193-510)$ & $278(195-516)$ & 0.02 \\
\hline Surgeon volume, median (IQR) & $69(47-105)$ & $70(46-104)$ & $69(47-105)$ & 0.01 \\
\hline General anesthesia & $7649(18.1)$ & $1024(18.2)$ & $6625(18.1)$ & 0.00 \\
\hline $\begin{array}{l}\text { Change in annual health care costs, } \\
2013 \text { dollars, median (IQR) }\end{array}$ & $-733(-2228$ to 2866$)$ & $501(-1587$ to 8417$)$ & $-865(-2292$ to 2071$)$ & 0.35 \\
\hline Readmission within $30 \mathrm{~d}$ & $130(0.3)$ & $58(1.0)$ & $72(0.2)$ & 0.11 \\
\hline $\begin{array}{l}\text { Note: IQR = interquartile range. } \\
\text { *Except where noted otherwise. } \\
\text { †Ontario Marginalization Index. } \\
\text { †Data not available for all patients. }\end{array}$ & & & & \\
\hline
\end{tabular}


Research

Table 2: Characteristics of eligible total knee arthroplasty recipients

\begin{tabular}{|c|c|c|c|c|}
\hline \multirow[b]{2}{*}{ Characteristic } & \multicolumn{3}{|c|}{ No. $(\%)$ of patients* } & \multirow[b]{2}{*}{$\begin{array}{l}\text { Standardized } \\
\text { difference }\end{array}$} \\
\hline & $\begin{array}{l}\text { All patients } \\
n=70725\end{array}$ & $\begin{array}{c}\text { Emergency } \\
\text { department visit } \\
n=11224\end{array}$ & $\begin{array}{c}\text { No emergency } \\
\text { department visit } \\
\quad n=59501\end{array}$ & \\
\hline Age, yr, median (IQR) & $67(61-74)$ & $68(61-75)$ & $67(61-74)$ & 0.09 \\
\hline \multicolumn{5}{|l|}{ Sex } \\
\hline Female & $43915(62.1)$ & $6543(58.3)$ & $37372(62.8)$ & 0.09 \\
\hline Male & $26810(37.9)$ & $4681(41.7)$ & $22129(37.2)$ & 0.09 \\
\hline Rural residence & 11505 (16.3) & $2563(22.8)$ & $8942(15.0)$ & 0.20 \\
\hline \multicolumn{5}{|l|}{ Ethnic concentration quintile†‡ } \\
\hline 1 (lowest) & $17345(24.7)$ & $3331(30.0)$ & $14014(23.7)$ & 0.14 \\
\hline 2 & $15976(22.7)$ & $2561(23.1)$ & $13415(22.7)$ & 0.01 \\
\hline 3 & $13634(19.4)$ & 2036 (18.3) & $11598(19.6)$ & 0.03 \\
\hline 4 & $11789(16.8)$ & $1616(14.5)$ & $10173(17.2)$ & 0.07 \\
\hline 5 (highest) & $11559(16.4)$ & $1564(14.1)$ & 9995 (16.9) & 0.08 \\
\hline \multicolumn{5}{|l|}{ Instability quintile†‡ } \\
\hline 1 (lowest) & $12143(17.3)$ & $1700(15.3)$ & $10443(17.6)$ & 0.06 \\
\hline 2 & $14062(20.0)$ & 2149 (19.3) & $11913(20.1)$ & 0.02 \\
\hline 3 & $14783(21.0)$ & $2372(21.4)$ & $12411(21.0)$ & 0.01 \\
\hline 4 & $14605(20.8)$ & $2526(22.7)$ & $12079(20.4)$ & 0.06 \\
\hline 5 (highest) & $14710(20.9)$ & $2361(21.3)$ & $12349(20.9)$ & 0.01 \\
\hline \multicolumn{5}{|l|}{ Dependency quintile†‡ } \\
\hline Lowest & $10123(14.4)$ & 1407 (12.7) & $8716(14.7)$ & 0.06 \\
\hline 2 & $11831(16.8)$ & $1702(15.3)$ & $10129(17.1)$ & 0.05 \\
\hline 3 & $13216(18.8)$ & $1975(17.8)$ & $11241(19.0)$ & 0.03 \\
\hline 4 & $14776(21.0)$ & 2466 (22.2) & $12310(20.8)$ & 0.03 \\
\hline 5 (highest) & $20357(29.0)$ & $3558(32.0)$ & $16799(28.4)$ & 0.08 \\
\hline \multicolumn{5}{|l|}{ Deprivation quintile†‡ } \\
\hline 1 (lowest) & $14270(20.3)$ & $2118(19.1)$ & $12152(20.5)$ & 0.04 \\
\hline 2 & $14722(20.9)$ & 2187 (19.7) & $12535(21.2)$ & 0.04 \\
\hline 3 & $14300(20.3)$ & $2339(21.1)$ & $11961(20.2)$ & 0.02 \\
\hline 4 & $14267(20.3)$ & $2328(21.0)$ & $11939(20.2)$ & 0.02 \\
\hline 5 (highest) & $12744(18.1)$ & 2136 (19.2) & 10608 (17.9) & 0.03 \\
\hline \multicolumn{5}{|l|}{ Income quintileł } \\
\hline 1 (lowest) & $12129(17.2)$ & 2152 (19.2) & $9977(16.8)$ & 0.06 \\
\hline 2 & $14566(20.6)$ & $2359(21.1)$ & $12207(20.6)$ & 0.01 \\
\hline 3 & $14652(20.8)$ & $2221(19.8)$ & $12431(20.9)$ & 0.03 \\
\hline 4 & $14458(20.5)$ & $2235(20.0)$ & $12223(20.6)$ & 0.02 \\
\hline 5 (highest) & $14773(20.9)$ & $2235(20.0)$ & $12538(21.1)$ & 0.03 \\
\hline \multicolumn{5}{|l|}{ Comorbidities } \\
\hline Asthma & $3268(4.6)$ & $630(5.6)$ & $2638(4.4)$ & 0.05 \\
\hline Coronary artery disease & $2186(3.1)$ & $425(3.8)$ & $1761(3.0)$ & 0.05 \\
\hline Congestive heart failure & $3271(4.6)$ & $701(6.2)$ & $2570(4.3)$ & 0.09 \\
\hline Chronic obstructive pulmonary disease & $13148(18.6)$ & $2521(22.5)$ & $10627(17.9)$ & 0.11 \\
\hline Counselled about smoking cessation & $3352(4.7)$ & $595(5.3)$ & $2757(4.6)$ & 0.03 \\
\hline Diabetes & $20508(29.0)$ & $3409(30.4)$ & $17099(28.7)$ & 0.04 \\
\hline Complicated diabetes & $3650(5.2)$ & $743(6.6)$ & $2907(4.9)$ & 0.07 \\
\hline Hypertension & 51267 (72.5) & $8358(74.5)$ & 42909 (72.1) & 0.05 \\
\hline Frailty & 3407 (4.8) & $635(5.7)$ & $2772(4.7)$ & 0.05 \\
\hline \multicolumn{5}{|l|}{ Charlson Comorbidity Index score } \\
\hline 0 & $50204(71.0)$ & $7596(67.7)$ & $42608(71.6)$ & 0.09 \\
\hline 1 & $13274(18.8)$ & $2252(20.1)$ & $11022(18.5)$ & 0.04 \\
\hline 2 & $4799(6.8)$ & $863(7.7)$ & $3936(6.6)$ & 0.04 \\
\hline$\geq 3$ & $2448(3.5)$ & $513(4.6)$ & 1935 (3.2) & 0.07 \\
\hline \multicolumn{5}{|l|}{ Admission characteristics } \\
\hline Hospital volume, median (IQR) & $429(315-607)$ & $427(311-597)$ & 429 (315-609) & 0.04 \\
\hline Surgeon volume, median (IQR) & $104(72-138)$ & $104(71-137)$ & $104(72-138)$ & 0.01 \\
\hline General anesthesia & $11470(16.2)$ & $1859(16.6)$ & $9611(16.2)$ & 0.01 \\
\hline $\begin{array}{l}\text { Change in annual health care costs, } \\
2013 \text { dollars, median (IQR) }\end{array}$ & $-586(-2356$ to 5248$)$ & $682(-1673$ to 8227$)$ & $-771(-2449$ to 4330$)$ & 0.30 \\
\hline Readmission within $30 \mathrm{~d}$ & $156(0.2)$ & $70(0.6)$ & $86(0.1)$ & 0.08 \\
\hline $\begin{array}{l}\text { Note: IQR = interquartile range. } \\
\text { ^Except where noted otherwise. } \\
\text { †Ontario Marginalization Index. } \\
\text { ‡Data not available for all patients. }\end{array}$ & & & & \\
\hline
\end{tabular}


Table 3: Reasons for emergency department visit within 30 days of surgery

\begin{tabular}{|lcc|}
\hline & \multicolumn{2}{c|}{ No. (\%) of patients } \\
\cline { 2 - 3 } Diagnosis & $\begin{array}{c}\text { Total hip } \\
\text { arthroplasty } \\
n=5640\end{array}$ & $\begin{array}{c}\text { Total knee } \\
\text { arthroplasty } \\
n=11224\end{array}$ \\
\hline Wound issue & $1614(28.6)$ & $2617(23.3)$ \\
\hline Concern about infection & $776(13.8)$ & $2316(20.6)$ \\
\hline Acute pain & $906(16.1)$ & $1777(15.8)$ \\
\hline Cardiovascular diagnosis & $501(8.9)$ & $1118(10.0)$ \\
\hline Gastrointestinal diagnosis & $487(8.6)$ & $1131(10.1)$ \\
\hline Possible thrombophlebitis & $402(7.1)$ & $623(5.6)$ \\
\hline $\begin{array}{l}\text { Family-practice-sensitive } \\
\text { condition }\end{array}$ & $308(5.5)$ & $578(5.1)$ \\
\hline Genitourinary disorder & $277(4.9)$ & $418(3.7)$ \\
\hline Preexisting medical issue & $191(3.4)$ & $197(1.8)$ \\
\hline Reaction to medications & $62(1.1)$ & $134(1.2)$ \\
\hline Miscellaneous & $116(2.0)$ & $315(2.8)$ \\
\hline
\end{tabular}

Table 4: Multivariable models predicting all-cause emergency department visit within $\mathbf{3 0}$ days of surgery

\begin{tabular}{|ll|}
\hline Variable & $\begin{array}{c}\text { Adjusted OR } \\
(95 \% \mathrm{Cl})\end{array}$ \\
\hline Total hip arthroplasty recipients & \\
\hline Age, yr & \\
\hline$<50$ v. 50-70 & $1.11(0.99-1.25)$ \\
\hline$>70$ v. 50-70 & $1.33(1.25-1.41)$ \\
\hline Male sex & $1.17(1.11-1.24)$ \\
\hline Chronic obstructive pulmonary disease & $1.28(1.20-1.38)$ \\
\hline Obesity & $1.30(1.16-1.46)$ \\
\hline Hypertension & $1.12(1.05-1.19)$ \\
\hline Rural residence & $1.48(1.38-1.59)$ \\
\hline C-statistic: 0.571 & \\
\hline Total knee arthroplasty recipients & \\
\hline Age, yr & \\
\hline < 50 v. 50-70 & $1.28(1.15-1.44)$ \\
\hline$>70$ v. 50-70 & $1.24(1.19-1.29)$ \\
\hline Male sex & $1.18(1.13-1.23)$ \\
\hline Chronic obstructive pulmonary disease & $1.26(1.19-1.32)$ \\
\hline Congestive heart failure & $1.28(1.17-1.39)$ \\
\hline Asthma & $1.25(1.14-1.37)$ \\
\hline Diabetes (complicated) & $1.21(1.11-1.32)$ \\
\hline Low income & $1.137-1.19)$ \\
\hline Rural residence & \\
\hline C-statistic: 0.578 & \\
\hline Note: Cl = confidence interval, OR = odds ratio. & \\
\hline
\end{tabular}

live far away from the hospital where they receive their surgery and may not have ready access to their surgeon. Furthermore, they may not always have timely access to primary care and may choose the emergency department for a last-minute assessment or reassurance. The needs of these patients might be potentially addressed with the implementation of a remote counselling system.

We found that most emergency department visits were related to pain or wound problems, consistent with the findings of studies in other regions. ${ }^{38,39} \mathrm{~A}$ recent survey showed that almost half of adults in Ontario reported going to the emergency department for a condition they thought could have been treated by a primary care provider. ${ }^{40}$ These nonemergent visits to emergency departments are an important contributor to long emergency department wait times. ${ }^{40}$ In 2015, the average length of an emergency department visit in urban areas in Ontario was over 8 hours. ${ }^{40}$ Given that more than 25000 hip and knee replacement operations are performed in Ontario alone each year, the impact of an emergency department presentation rate of $15 \%$ following joint replacement is considerable. A concerted effort to identify patients at increased risk, coupled with an intervention to minimize this rate, could have a substantial effect on emergency department wait times, possibly resulting in an improvement in patient care and a reduction in burnout for emergency department health care providers.

We were able to identify only modest predictors for an emergency department visit because our models had an area under the curve of about 0.57 . This indicates that our models are only slightly better than chance at predicting emergency department visits. This is likely a shortfall from administrative data since our analysis is missing information on level of support at home and other relevant factors. We are currently conceptualizing future research that will use patient-level data, including qualitative interviews with patients, about their experiences after discharge following total joint arthroplasty to better understand the reasons for subsequent emergency visits.

\section{Limitations}

This study has several limitations. These pertain mainly to data that are unavailable in the administrative databases used to conduct the study, including patient body mass index, smoking status, alcohol use and level of support of home, all of which may affect the risk of early complications and presentation to the emergency department. We attempted to mitigate this limitation by measuring neighbourhood income quintile and marginalization indices, characteristics that correlate with the prevalence of alcohol abuse and obesity. ${ }^{41-46}$ In addition, as we relied primarily on diagnosis codes to identify the reasons for presenting to the emergency department, we did not have the data clarity to understand why patients visit an emergency department or to identify all predictive factors accurately. Finally, we did not have the time of the emergency department visit available - this variable is likely related to the availability of other care resources. 


\section{Conclusion}

About 1 in 7 patients in Ontario presented to an emergency department within 30 days of primary hip or knee replacement, with almost half presenting at an institution different from where they had their surgery. Most of the reasons for presentation were related to the surgical wound and pain. Fewer than $1 \%$ of patients who presented to an emergency department required readmission, and the reasons for presentation were those that can conceivably be managed remotely. Future studies should explore more specific data that may be associated with emergency department presentation and characterize patients' reasons for presenting to the emergency department.

\section{References}

1. Hospital Readmissions Reduction Program (HRRP). Baltimore: Centers for Medicare \& Medicaid Services (CMS); 2018. Available: https://www.cms. gov/medicare/quality-initiatives-patient-assessment-instruments/value-based -programs/hrrp/hospital-readmission-reduction-program.html (accessed 2019 Oct. 1).

2. Ali AM, Loeffler M, Aylin P, et al. Factors associated with 30-day readmission after primary total hip arthroplasty. ZAMA Surg 2017;152:e173949.

3. Kelly MP, Prentice H, Wang W, et al. Reasons for ninety-day emergency visits and readmissions after elective total joint arthroplasty: results from a US integrated healthcare system. F Arthroplasty 2018;33:2075-81.

4. Saleh A, Faour M, Sultan A, et al. Emergency department visits within thirty days of discharge after primary total hip arthroplasty: a hidden quality measure. 7 Arthroplasty 2019;34:20-6.

5. All-cause readmission to acute care and return to the emergency department. Ottawa: Canadian Institute for Health Information; 2012. Available: https:// secure.cihi.ca/free_products/Readmission_to_acutecare_en.pdf (accessed 2019 Oct. 1).

6. Juurlink D, Preyra C, Croxford R, et al. Canadian Institute for Health Information Discharge Abstract Database: a validation study. Toronto: Institute for Clinical Evaluative Sciences; 2006.

7. Agabiti N, Picciotto S, Cesaroni G, et al. The influence of socioeconomic status on utilization and outcomes of elective total hip replacement: a multicity population-based longitudinal study. Int 7 Qual Health Care 2007;19:37-44.

8. Santaguida PL, Hawker GA, Hudak PL, et al. Patient characteristics affecting the prognosis of total hip and knee joint arthroplasty: a systematic review. Can 7 Surg 2008;51:428-36.

9. Kralj B. Measuring "rurality" for purposes of health care planning: an empirical measure for Ontario. Toronto: Ontario Medical Association; 2005.

10. Continuity of care with family medicine physicians: why it matters. Ottawa: Canadian Institute for Health Information; 2015. Available: https://secure.cihi. ca/free_products/UPC_ReportFINAL_EN.pdf (accessed 2019 Oct. 1).

11. Gershon AS, Guan J, Wang C, et al. Trends in asthma prevalence and incidence in Ontario, Canada, 1996-2005: a population study. Am 7 Epidemiol 2010;172:728-36.

12. Ko DT, Mamdani M, Alter DA. Lipid-lowering therapy with statins in highrisk elderly patients: the treatment-risk paradox. FAMA 2004;291:1864-70.

13. Hux JE, Ivis F, Flintoft V, et al. Diabetes in Ontario: determination of prevalence and incidence using a validated administrative data algorithm. Diabetes Care 2002;25:512-6.

14. Tu K, Campbell NR, Chen ZL, et al. Accuracy of administrative databases in identifying patients with hypertension. Open Med 2007;1:e18-26.

15. Gershon AS, Wang C, Guan J, et al. Identifying individuals with physician diagnosed COPD in health administrative databases. COPD 2009;6:388-94.

16. Deyo RA, Cherkin DC, Ciol MA. Adapting a clinical comorbidity index for use with ICD-9-CM administrative databases. 7 Clin Epidemiol 1992;45:613-9.

17. Quan H, Sundararajan V, Halfon P, et al. Coding algorithms for defining comorbidities in ICD-9-CM and ICD-10 administrative data. Med Care 2005; 43:1130-9.

18. Weiner JP, Abrams C. The Johns Hopkins ACG® System: technical reference guide. Version 10.0. Baltimore: Johns Hopkins University; 2011.

19. Schedule of benefits: physician services under the Health Insurance Act (June 3, 2008). Toronto: Ministry of Health and Long-Term Care; 2008. Available: www.health.gov.on.ca/en/pro/programs/ohip/sob/physserv/sob_physician_services_ 20080603.pdf (accessed 2019 Oct. 1).

20. Reducing gaps in health: a focus on socio-economic status in urban Canada. Ottawa: Canadian Institute for Health Information; 2008.

21. Glazier RH, Badley E, Gilbert J, et al. The nature of increased hospital use in poor neighbourhoods: findings from a Canadian inner city. Can 7 Public Health 2000;91:268-73.
22. Matheson FI, van Ingen T. 2016 Ontario Marginalization Index: user guide. Toronto: St. Michael's Hospital and Public Health Ontario; 2018. Available: https://www.publichealthontario.ca/-/media/documents/on-marg-userguide.pdf? la=en (accessed 2019 Oct. 1).

23. Matheson F, Dunn J, Smith K, et al. Ontario Marginalization Index (ON-Marg): user guide. Toronto: Centre for Research in Inner City Health, St. Michael's Hospital; 2011.

24. Bai Y, Santos G, Wodchis W. Cost of public bealth services for Ontario residents injured as a result of a motor vehicle accident. Applied bealth research question series. Toronto: Health System Performance Research Network; 2016. Available: www.fairassociation.ca/wp-content/uploads/2016/03/HSPRN-AHRQ -MVA-Report-2016-FINAL1.pdf (accessed 2019 Oct. 1).

25. Tu JV, Sykora K, Naylor CD. Assessing the outcomes of coronary artery bypass graft surgery: How many risk factors are enough? Steering Committee of the Cardiac Care Network of Ontario. 7 Am Coll Cardiol 1997;30:1317-23.

26. Medical Advisory Secretariat. Total knee replacement: an evidence-based analysis. Ont Health Technol Assess Ser 2005;5:1-51.

27. Hawker GA, Badley EM, Croxford R, et al. A population-based nested casecontrol study of the costs of hip and knee replacement surgery. Med Care 2009; 47:732-41.

28. Wodchis WP, Bushmeneva K, Nikitovic M, et al. Guidelines on person-level costing using administrative databases in Ontario. Working Paper series vol. 1. Toronto: Health System Performance Research Network; 2013.

29. Chen A, Bushmeneva K, Zagorski B, et al. Direct cost associated with acquired brain injury in Ontario. BMC Neurol 2012;12:76.

30. Nikitovic M, Wodchis WP, Krahn MD, et al. Direct health-care costs attributed to hip fractures among seniors: a matched cohort study. Osteoporos Int 2013;24:659-69.

31. Munce SEP, Wodchis WP, Guilcher SJT, et al. Direct costs of adult traumatic spinal cord injury in Ontario. Spinal Cord 2013;51:64-9.

32. Rosella LC, Fitzpatrick T, Wodchis WP, et al. High-cost health care users in Ontario, Canada: demographic, socio-economic, and health status characteristics. BMC Health Serv Res 2014;14:532.

33. Tanuseputro P, Wodchis WP, Fowler R, et al. The health care cost of dying: a population-based retrospective cohort study of the last year of life in Ontario, Canada. PLoS One 2015;10:e0121759.

34. Rosella LC, Lebenbaum M, Fitzpatrick T, et al. Impact of diabetes on healthcare costs in a population-based cohort: a cost analysis. Diabet Med 2016;33: 395-403.

35. Dumestre DO, Fraulin FOG. Balancing the need for clinical photography with patient privacy issues: the search for a secure smartphone application to take and store clinical photographs. Plast Surg (Oakv) 2017;25:255-60.

36. Using electronic communications, protecting privacy. Ottawa: Canadian Medical Protective Association; revised 2016 Jan. Available: https://www. cmpa-acpm.ca/en/advice-publications/browse-articles/2013/using-electronic -communications-protecting-privacy (accessed 2019 Oct. 1).

37. Dawson H, Zinck G. CIHI survey: ED spending in Canada: a focus on the cost of patients waiting for access to an in-patient bed in Ontario. Healthc Q 2009; $12: 25-8$.

38. Finnegan MA, Shaffer R, Remington A, et al. Emergency department visits following elective total hip and knee replacement surgery: identifying gaps in continuity of care. F Bone Foint Surg Am 2017;99:1005-12.

39. Kelly MP, Prentice HA, Wang W, et al. Reasons for ninety-day emergency visits and readmissions after elective total joint arthroplasty: results from a US integrated healthcare system. F Arthroplasty 2018;33:2075-81.

40. Under pressure: emergency department performance in Ontario. Toronto: Health Quality Ontario; 2017. Available: http://underpressure.hqontario.ca/ (accessed 2019 Oct. 1)

41. Sanmartin C, Ng E, Blackwell D, et al. Foint Canada/United States Survey of Health, 2002-03. Ottawa: Statistics Canada; 2004. Cat no 82M0022XIE.

42. Kralj B. Measuring "rurality" for purposes of health-care planning: an empirical measure for Ontario. Ont Med Rev 2000;(Oct):37-40.

43. Pincus D, Ravi B, Wasserstein D, et al. Association between wait time and 30-day mortality in adults undergoing hip fracture surgery. $7 A M A$ 2017;318: 1994-2003.

44. How healthy are rural Canadians? An assessment of their health status and health determinants. Ottawa: Canadian Institute for Health Information; 2006.

45. White H, Matheson F, Moineddin R, et al. Neighbourhood deprivation and regional inequalities in self-reported health among Canadians: Are we equally at risk? Health Place 2011;17:361-9.

46. Matheson FI, Moineddin R, Glazier R. The weight of place: a multilevel analysis of gender, neighborhood material deprivation, and body mass index among Canadian adults. Soc Sci Med 2008;66:675-90.

Affiliations: Division of Orthopaedic Surgery (Ravi), Department of Surgery and Department of Medicine (Redelmeier), University of Toronto; Division of Orthopaedic Surgery (Ravi), Sunnybrook Health Sciences Centre; ICES (Ravi, Austin, Paterson, Aktar, Redelmeier); Division of Orthopaedic Surgery (Leroux), Toronto Western Hospital; Evaluative Clinical Sciences (Austin, Redelmeier), Sunnybrook Research Institute, Toronto, Ont. 
Contributors: Bheeshma Ravi and Donald Redelmeier interpreted the data and revised the manuscript critically for important intellectual content. All of the authors contributed to the study conception and design, analyzed the data, drafted the manuscript, approved the final version to be published and agreed to be accountable for all aspects of the work.

Funding: This study was supported by a grant from the Canadian Institutes of Health Research and by ICES, a nonprofit research institute funded by the Ontario Ministry of Health and Long-Term Care. Peter Austin is supported in part by a Mid-Career Investigator Award from the Heart and Stroke Foundation.
Disclaimer: Parts of this material are based on data and information compiled and provided by the Canadian Institute for Health Information. The opinions, results and conclusions reported in this paper are those of the authors and are independent from the data providers and funding sources. No endorsement by the Canadian Institutes of Health Research, ICES or the Ontario Ministry of Health and Long-Term Care is intended or should be inferred.

Supplemental information: For reviewer comments and the original submission of this manuscript, please see www.cmajopen.ca/content/8/1/ E26/suppl/DC1. 